\title{
Chronic Alcohol Ingestion in Rats Alters Lung Metabolism, Promotes Lipid Accumulation, and Impairs Alveolar Macrophage Functions
}

\author{
Freddy Romero ${ }^{1}$, Dilip Shah ${ }^{1}$, Michelle Duong ${ }^{1}$, William Stafstrom ${ }^{1}$, Jan B. Hoek ${ }^{2}$, Caleb B. Kallen ${ }^{4}$, \\ Charles H. Lang ${ }^{3}$, and Ross Summer ${ }^{1}$ \\ ${ }^{1}$ Center for Translational Medicine, ${ }^{2}$ Department of Pathology, Anatomy, and Cell Biology, and ${ }^{4}$ Department of Obstetrics and \\ Gynecology, Thomas Jefferson University, Philadelphia, Pennsylvania; and ${ }^{3}$ Department of Cellular and Molecular Physiology, Penn \\ State College of Medicine, Hershey, Pennsylvania
}

\begin{abstract}
Chronic alcoholism impairs pulmonary immune homeostasis and predisposes to inflammatory lung diseases, including infectious pneumonia and acute respiratory distress syndrome. Although alcoholism has been shown to alter hepatic metabolism, leading to lipid accumulation, hepatitis, and, eventually, cirrhosis, the effects of alcohol on pulmonary metabolism remain largely unknown. Because both the lung and the liver actively engage in lipid synthesis, we hypothesized that chronic alcoholism would impair pulmonary metabolic homeostasis in ways similar to its effects in the liver. We reasoned that perturbations in lipid metabolism might contribute to the impaired pulmonary immunity observed in people who chronically consume alcohol. We studied the metabolic consequences of chronic alcohol consumption in rat lungs in vivo and in alveolar epithelial type II cells and alveolar macrophages (AMs) in vitro. We found that chronic alcohol ingestion significantly alters lung metabolic homeostasis, inhibiting AMP-activated protein kinase, increasing lipid synthesis, and suppressing the expression of genes essential to metabolizing fatty acids (FAs). Furthermore, we show that these metabolic alterations promoted a lung phenotype that is reminiscent of alcoholic fatty liver and is characterized by marked accumulation of triglycerides and free FAs within distal airspaces, AMs, and, to a lesser extent, alveolar epithelial type II cells. We provide evidence that the metabolic alterations in alcohol-exposed rats are mechanistically linked to immune impairments in the alcoholic lung: the elevations in FAs alter AM phenotypes and suppress both phagocytic functions and agonist-induced inflammatory responses. In summary, our
\end{abstract}

work demonstrates that chronic alcohol ingestion impairs lung metabolic homeostasis and promotes pulmonary immune dysfunction. These findings suggest that therapies aimed at reversing alcohol-related metabolic alterations might be effective for preventing and/or treating alcohol-related pulmonary disorders.

Keywords: chronic alcohol ingestion; AMP-activated protein kinase; surfactant lipids; macrophage

\section{Clinical Relevance}

Chronic alcohol abuse is a risk factor for bacterial pneumonia and acute respiratory distress syndrome; the molecular mechanisms underlying this association are not understood. Our work demonstrates that chronic alcohol exposure induces significant metabolic changes in the lung, including marked accumulation of triglycerides and free fatty acids within distal airspaces and alveolar macrophages (AMs). Furthermore, we provide evidence linking these lipid abnormalities to phenotypic and functional impairments in AMs, suggesting that these metabolic disturbances may contribute to the pathogenesis of alcohol-related inflammatory lung diseases. Together, these observations have broad implications for studying the effects of alcohol on lung homeostasis and immunity, and may be relevant to the pathogenesis of other inflammatory pulmonary disorders.

(Received in original form March 21, 2014; accepted in final form June 11, 2014)

This work was supported by National Institutes of Health grants R01HL105490 and R37 AA11290.

Correspondence and requests for reprints should be addressed to Ross Summer, M.D., Center for Translational Medicine, Thomas Jefferson University, Philadelphia, PA 19107. E-mail: ross.summer@jefferson.edu

This article has an online supplement, which is accessible from this issue's table of contents at www.atsjournals.org

Am J Respir Cell Mol Biol Vol 51, Iss 6, pp 840-849, Dec 2014

Copyright () 2014 by the American Thoracic Society

Originally Published in Press as DOI: 10.1165/rcmb.2014-01270C on June 18, 2014

Internet address: www.atsjournals.org 
Chronic ethanol (EtOH) consumption injures all tissues, but certain organs, such as the liver, heart, and brain, are particularly susceptible. Although the lung is not considered to be the most important target of EtOH-mediated tissue injury, pulmonary toxicity is well documented $(1,2)$. Heavy $\mathrm{EtOH}$ consumption disrupts various biological processes in the lung, including mucociliary clearance, oxidant-antioxidant balance, and alveolar macrophage (AM) function (3-5). Moreover, chronic EtOH abuse predisposes to the development of various inflammatory lung disorders, including infectious pneumonia and acute respiratory distress syndrome, and clinical outcomes for these conditions are worse in patients that chronically consume EtOH (6-8).

Research over the past several decades has focused on the inflammatory nature of EtOH-induced lung disorders, and has explored the immune mechanisms underlying susceptibility to these diseases (9-11). This mechanistic focus in the lung contrasts the intense study of the effects of EtOH on metabolic homeostatic processes in other organs, such as the liver. It has been shown that EtOH induces significant metabolic disturbances in the liver, and these perturbations are thought to contribute to the pathogenesis of EtOHrelated liver diseases, such as alcoholic fatty liver disease, steatohepatitis, and cirrhosis (12-14).

To date, little is known regarding the effects of EtOH on lung metabolism. This is particularly surprising when one considers that the lung, like the liver, synthesizes lipids de novo. In the lung, these lipids are synthesized by specialized cells that reside within distal airspaces called type II alveolar epithelial (AEII) cells $(15,16)$, and their production is required for generating the surfactant monolayer that is critical for reducing surface tension and protecting the underlying respiratory epithelium. Surfactant lipids are comprised principally ( $\sim 85 \%)$ of phospholipids (PLs), whereas cholesterol, triglycerides (TG) and free fatty acids (FAs) collectively represent only 10-15\% of the total surfactant lipid pool $(15,17)$.

Studies investigating the effects of EtOH on surfactant lipid homeostasis have focused principally on PL production. For example, Guidot and colleagues (18) showed that EtOH-fed rats have reduced incorporation of $[3 \mathrm{H}]$ choline into PLs in AEII cells. Similarly, Wagner and Heinemann (19) found that prefeeding rats for 3 days with low concentrations of $\mathrm{EtOH}$ significantly decreased precursor incorporation into PLs. Although an anticipated reduction in overall $\mathrm{PL}$ concentrations would be expected based upon these studies, total and fractionated forms of PLs have not been shown to be significantly decreased in chronically EtOH-exposed lungs, suggesting that the lung possesses mechanisms for limiting excursions in PL levels in response to chronic EtOH ingestion. To date, studies examining the effects of $\mathrm{EtOH}$ on other lipid species in the lung are limited, though existing evidence suggests that cholesterol
A

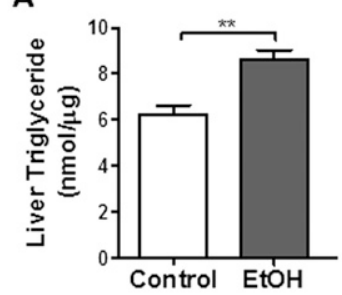

C

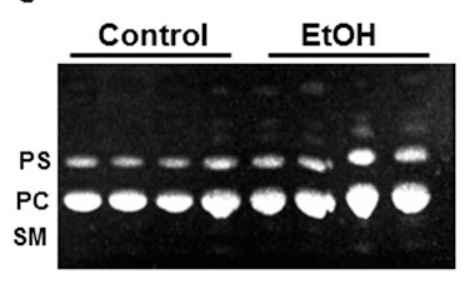

$\mathbf{F}$

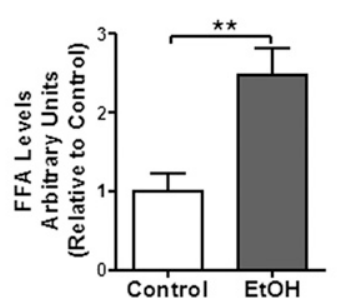

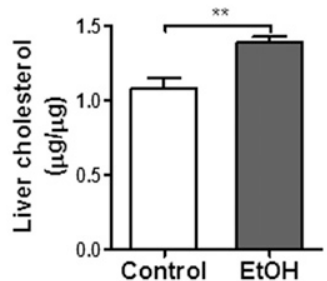

D

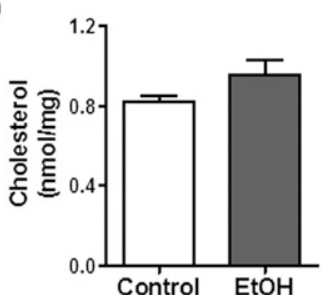

G

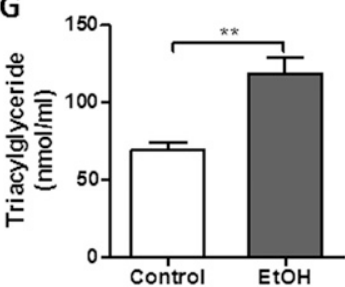

B

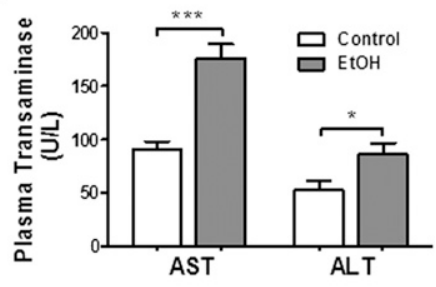

E

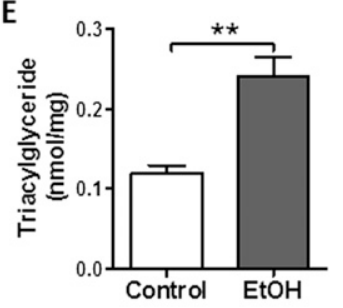

H

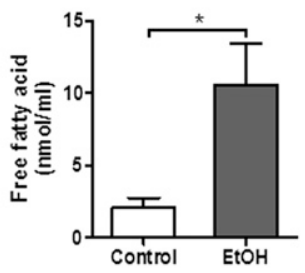

Figure 1. Chronic ethanol (EtOH) ingestion induces lipid accumulation in the liver and lung. (A) Chronic EtOH ingestion increased triglycerides (TGs) and cholesterol levels in the liver ( $n=4$ each group, ${ }^{\star \star} P<0.01$ versus control group). (B) Plasma aspartate and alanine aminotransferase levels are elevated in chronic EtOH fed rats ( $n=10$ each group; ${ }^{\star} P<0.05$, ${ }^{* \star \star} P<0.001$ versus control group). (C) Chronic EtOH ingestion did not significantly increase total phospholipids (PLs), phosphotidylcholine (PC), or phosphotidylserine (PS) in whole lung $(n=10)$. The image is representative of two different TLC. $\mathrm{SM}$, sphingomyelin. (D) Chronic EtOH ingestion did not significantly increase total cholesterol levels in whole lung $(n=10)$. ( $E-F)$ Chronic EtOH ingestion enhanced TG and fatty acid (FA) levels in whole lung $\left(n=10,{ }^{\star *} P<0.01\right.$ versus control group). (G-H) Chronic EtOH ingestion enhanced TG $\left(n=10\right.$, ${ }^{* *} P<0.01$ versus control group) and FA levels in bronchoalveolar lavage (BAL) $\left(n=10,{ }^{\star} P<0.05\right.$ versus control group). All data are expressed as means ( \pm SE). The statistical significance was assessed using a Student's unpaired $t$ test. 
and cholesterol esters are not significantly affected, whereas TGs appear to be markedly increased in response to chronic $\mathrm{EtOH}$ exposure $(17,19)$. The molecular mechanisms mediating TG accumulation in EtOH-exposed lungs, and the functional significance of these biochemical changes, remain unknown.

Because chronic EtOH consumption is known to alter lipid homeostasis in the liver, we hypothesized that similar metabolic disturbances would be observed in the lung, and that these changes might, at least in part, contribute to development of the immune impairments observed in response to chronic alcohol consumption. Consistent with this hypothesis, we observed decreased AMPactivated protein kinase (AMPK) activation and increased lipid synthesis in EtOHexposed lungs and in cultured AEII cells.

These metabolic changes were associated with marked lipid accumulation in EtOH-affected lungs, a phenotype for which we have now coined the expression, "the alcoholic fatty lung." Quantitative lipid analyses further demonstrated that lipid accumulation is largely attributable to increases in TGs and FAs, whereas PLs and cholesterol fractions are not significantly changed in response to chronic EtOH consumption. Furthermore, we provide evidence suggesting that lipid accumulation is causally related to immune impairments in the alcoholic lung by altering AM phenotype and function.

\section{Materials and Methods}

\section{Rat Model of Chronic Alcohol Ingestion}

Male Sprague-Dawley rats (Charles River Laboratory, Wilmington, MA) were fed with Lieber-DeCarli liquid (36\% calories from EtOH) or nonalcoholic isocaloric control liquid diet (Research Diets, New Brunswick, NJ) for 4 months (20). Information on body weight and plasma EtOH levels are provided in Table E1 in the online supplement. Animal protocols were reviewed and approved by the Institutional Animal Care and Use Committees at Penn State College of Medicine and Thomas Jefferson University, and adhered to the National Institutes of Health guidelines for the use of experimental animals.

\section{Bronchoalveolar Lavage Recovery and Fractionation}

Bronchoalveolar lavage (BAL), total cell counts, and differential cell counts were performed as previously described (21).

\section{AEII Cell Culture and Treatment}

Rat AEII cell line (L2 cells) was obtained from ATCC (Manassas, VA) and cultured according to company protocol. See the Materials and Methods section in the online supplement for details.

\section{Lipid Droplet Determination}

See the Materials and Methods section in the online supplement for details.

\section{Lipid Extraction and Analysis}

Total lipids were extracted from BAL fluid, L2 cells, and lung tissue using a modified method of Bligh and Dyer, using chloroform/methanol (2:1) (22). See the Materials and Methods section in the online supplement for further details regarding the extraction methods and separation protocols used in our studies.

\section{NR8383 Cell Culture and LPS Stimulation}

Rat AMs NR8383 cells were purchased from ATCC. Cells were cultured in RPMI

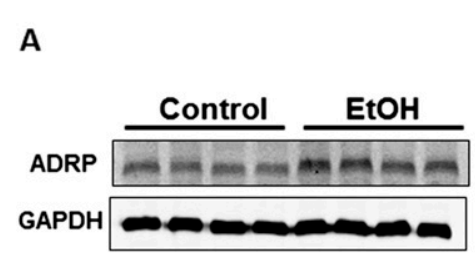

B

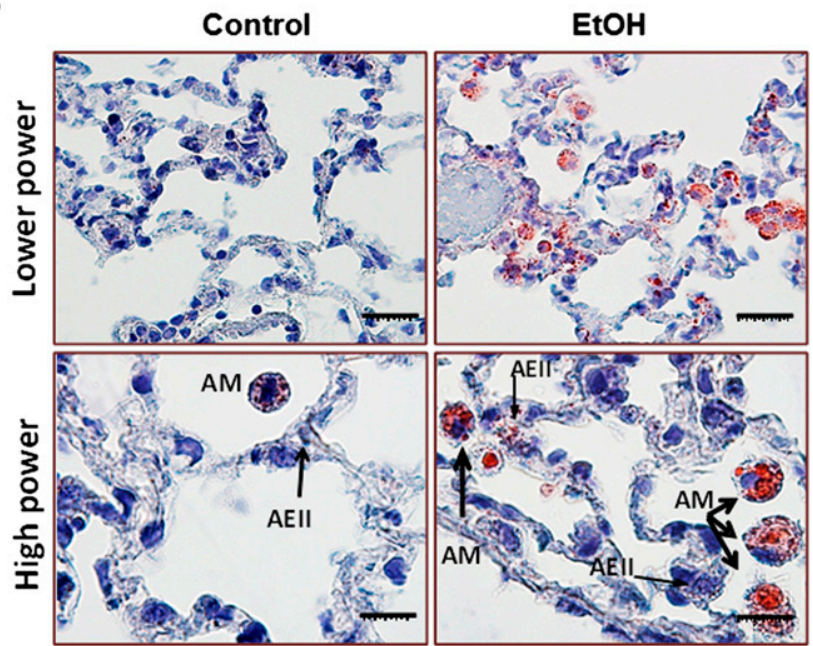

Figure 2. Chronic EtOH ingestion induces alveolar macrophage (AM) lipid accumulation. (A) Western blot analysis for adipose differentiation-related protein (ADRP) in lungs from chronic EtOH-fed rats $(n=$ 8). The image is representative of at least two different Western blots. Densitometry measurements showing increased ADRP expression in the lung from chronic $\mathrm{EtOH}$-fed rats ${ }^{\star \star} P<0.01$ versus control group). All data are expressed as means ( \pm SE). The statistical significance was assessed using a Student's unpaired $t$ test. $(B)$ Oil Red $O$ staining of the lung demonstrates massive neutral accumulation in $\mathrm{AMs}$ from $\mathrm{EtOH}$ fed rats while a more modest accumulation of lipid droplets was observed in alveolar epithelial type II (AEII) cells (thin arrows). 
A

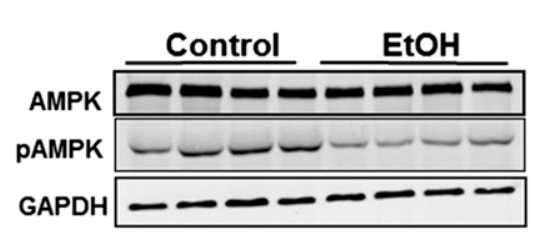

B

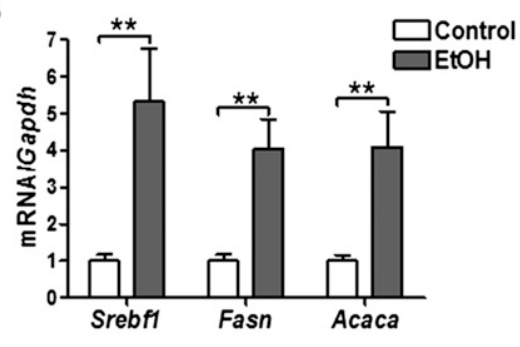

D

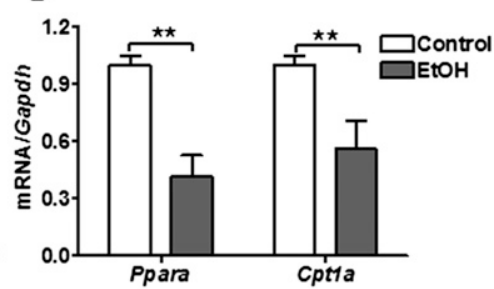

Figure 3. Chronic EtOH ingestion promotes metabolic changes in the lung. (A) Chronic EtOH ingestion decreased AMP-activated protein kinase (AMPK) activation, but had no effect on total protein levels in the lung $\left(n=8,{ }^{\star \star} P<0.01\right.$ versus control group). The image is representative of at least two different blots. (B) Transcript levels for sterol regulatory-element binding protein 1 (Srebf1), FA synthase (Fasn), and acetyl coenzyme A (CoA) carboxylase (Acaca) are increased in the lung after chronic EtOH consumption $\left(n=8,{ }^{\star \star} P<0.01\right.$ versus control group). (C) Protein expression for diacylglycerol O-acyltransferase 1 (DGAT1), SREBF1, FASN, and ACACA levels are increased in the lung after chronic EtOH ingestion $\left(n=8,{ }^{\star} P<0.05,{ }^{\star} * P<0.01\right.$, ${ }^{* \star \star} P<0.001$ versus Control group). The image is representative of two different Western blots. (D) Peroxisome proliferator-activated receptor $\alpha(P p a r a)$ and carnitine palmitoyltransferase I (Cpt1a) transcript levels are decreased in the lung after chronic EtOH intake $\left(n=8,{ }^{\star \star} P<0.01\right.$ versus control group). Data are expressed as means ( $\pm \mathrm{SE}$ ). The statistical significance was assessed using a Student's unpaired $t$ test.

$\alpha$ (Ppara), carnitine palmitoyltransferase I (Cpt1a), ATP-binding cassette subfamily G member 1 (Abcg1), ATPbinding cassette sub-family A member 1 (Abca1), Cd36, Msr1, Tgf- $\beta 1, A r g 1$, and Chi3l3. The housekeeping genes, Gapdh and Hprt1, were used for normalization. See the Materials and Methods section in the online supplement for specific details regarding quantitative RT-PCR and for the oligonucleotide sequences used in these studies.

\section{Western Blot Analysis}

Western blot analysis was performed for AMPK, phosphorylated-AMPK, ACACA, acetyl-CoA synthase, FA synthase, glyceraldehyde 3-phosphate dehydrogenase, adipose differentiation-related protein, SREBF1, diacylglycerol O-acyltransferase (DGAT) 1 and cytochrome P450 2E1 (CYP2E1); detailed protocols are described in the Materials and Methods section in the online supplement.

\section{Macrophage Phagocytosis Assay}

Details are described in the Materials AND Methods section in the online supplement.

\section{Alcohol Dehydrogenase Activity}

Alcohol dehydrogenase (ADH) activity in lung tissue and cultured cells was quantified using a commercially available kit (Bio Vision, Montain View, CA) according to the manufacturer's instructions.

\section{Plasma Measurements}

See the Materials and Methods section in the online supplement for details regarding measurements of plasma alanine and aspartate aminotransferases and $\mathrm{EtOH}$ concentration.

\section{Statistical Analysis}

Statistical analyses were performed using GraphPad Prism 5.0 software (GraphPad Inc., La Jolla, CA). Two-group comparisons were analyzed by unpaired Student's $t$ test, and multiple-group comparisons were performed by one-way ANOVA followed by Tukey's post hoc analysis. Statistical significance was achieved when $P$ was less than 0.05 at a $95 \%$ confidence interval.

\section{Results}

\section{Chronic Alcohol Ingestion Promotes Lipid Accumulation in the Lung}

To determine the effects of chronic EtOH ingestion on lipid homeostasis, we first assessed whether hepatic steatosis developed in our model. As shown in Figure 1A, we detected a significant increase in TG and cholesterol levels in livers from chronic EtOH-exposed rats. Moreover, these metabolic changes were associated with an increase in plasma alanine and aspartate aminotransferases (Figure 1B), indicating that chronic EtOH consumption induced hepatocellular injury in our model.

Next, we studied the effects of chronic EtOH exposure on lung lipid homeostasis. When comparing control and $\mathrm{EtOH}$-exposed whole lungs, we did not detect differences in total PLs, phosphotidylcholine, or phosphotidylserine (Figure 1C). Similarly, total cholesterol levels in whole lung were not significantly affected by chronic EtOH ingestion (Figure 1D), as previously described (17). We did, however, observe a marked increase in TGs and FAs in whole lung (Figures 1E and $1 F$ ) and in BAL fluid (Figures $1 G$ and $1 \mathrm{H})$ after chronic EtOH ingestion. These findings were associated with increased adipose differentiation-related protein (a.k.a. Plin2) protein content (Figure 2A), a marker of increased intracellular TG mobilization and storage. Moreover, Oil Red $\mathrm{O}$ staining of the lung detected massive accumulation of neutral lipids (red color) in AMs, whereas more subtle increases in lipid droplets were observed in other parenchymal cells, including AEII cells (Figure 2B). 


\section{Chronic Alcohol Ingestion Promotes Lipid Synthesis in the Lung}

To assess whether TG and FA accumulation resulted from metabolic disturbances that promote lipid synthesis, we evaluated the activation state of AMPK in the lungs of control and EtOH-fed rats. AMPK is a serine-threonine kinase that regulates substrate use in cells. Inhibition of AMPK by dephosphorylation at Thr172 is associated with activation of anabolic pathways (such as lipid synthesis) and suppression of catabolic processes (such as the breakdown of FAs) (23). Consistent with this functional paradigm, AMPK phosphorylation was significantly decreased in the lung after chronic EtOH consumption, and this was associated with up-regulation of transcripts and proteins for several key factors involved in lipid synthesis, including Srebf1, FA synthase (Fasn), Acaca, and Dgat1 (Figures 3A-3C). In addition, we observed decreased mRNA expression of Ppara and carnitine palmitoyltransferase I (Cpt1a) in lungs from chronic EtOH-fed rats (Figure 3D), suggesting that impaired breakdown of FAs may also contribute to TG and FA accumulation.

\section{EtOH Modifies AEII Cell Metabolism by Inhibiting AMPK Activation and Promoting Lipid Synthesis}

Our data suggested that alcohol induces lipid synthesis in the lung. To determine whether this might be a direct effect of alcohol on AEII cells, we tested whether EtOH directly modifies metabolism in L2 cells, a model cell system that demonstrates many features of alveolar epithelial cells. We detected marked intracellular TG accumulation in response to both low $(0.05 \%)$ and high $(0.1 \% \mathrm{vol} / \mathrm{vol})$ concentrations of EtOH after 48- or 72-hour exposure (Figure 4A). Similarly, Oil Red $\mathrm{O}$ staining detected increased lipid droplet formation in L2 cells after EtOH exposure (Figure 4B), consistent with our histological observations of increased intracellular lipids in AEII cells from chronic EtOH-fed rats. In contrast, intracellular lipid accumulation was not detected in rat NR8383 AMs after culture in $\mathrm{EtOH}$, suggesting that in vivo lipid accumulation in macrophages might result from enhanced lipid uptake rather than from increased production (data not shown). As observed in the alcohol-exposed lung, we found that increased TG accumulation in L2 cells was associated with broad metabolic changes, including decreased activation of AMPK, increased expression of SREBF1, and increased expression of the lipid-synthesizing enzyme, acetyl-CoA synthase (Figures 4C and 4D). However, levels of Ppara and Cpt1a transcripts were not significantly altered in response to EtOH (data not shown), suggesting that TG accumulation in $\mathrm{L} 2$ cells occurs primarily from increased lipid synthesis. Together, these findings indicate that EtOH significantly modifies AEII cell lipid metabolism, leading to lipid accumulation in macrophages, AEII cells, and the extracellular air spaces of the chronic EtOH-exposed lung.
A

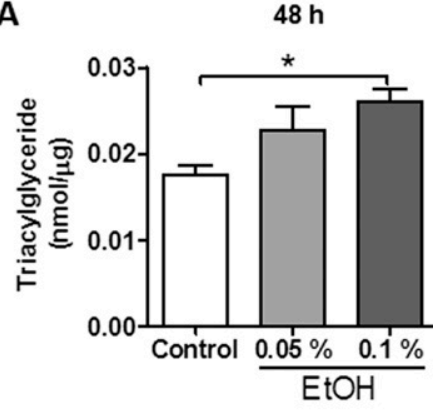

C

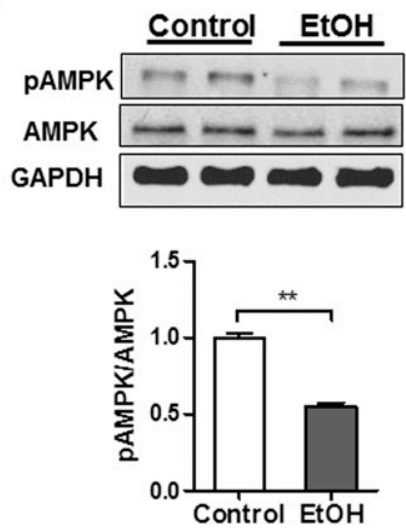

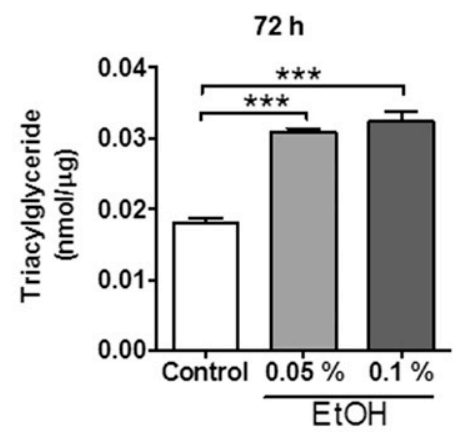

B

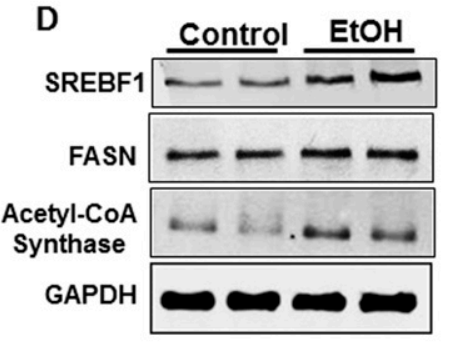

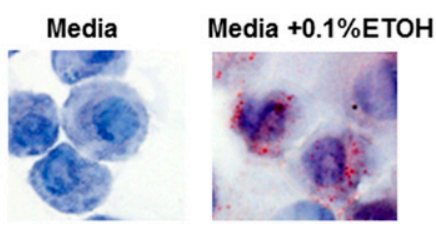

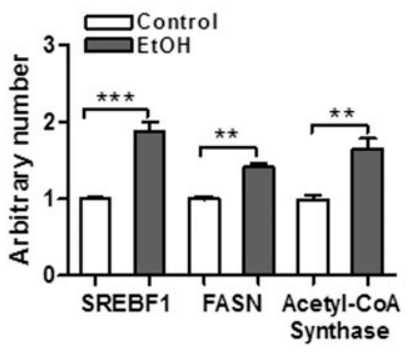

Figure 4. EtOH alters metabolic homeostasis in AEll cells leading to intracellular TG accumulation. (A) Rat L2 AEll cells accumulate TGs when cultured in 0.05 or $0.1 \% \mathrm{EtOH}$ for either 48 or 72 hours $\left(n=6,{ }^{*} P<0.05\right.$, ${ }^{\star \star \star} P<0.001$ versus control group). (B) Oil Red O staining of $\mathrm{L} 2$ cells demonstrated increased lipid droplet formation in response to $\mathrm{EtOH}$. (C) EtOH decreases AMPK activation (i.e., decreased phosphorylation) in $\mathrm{L} 2$ cells $\left(n=6\right.$, ${ }^{\star \star} P<0.01$ versus control group). The image is representative of three different blots. (D) EtOH enhances protein content of SREBF1 and acetyl-CoA synthase in L2 cells $\left(n=6,{ }^{\star \star} P<0.01,{ }^{\star \star \star} P<0.001\right)$. Data are expressed as means $( \pm \mathrm{SE})$. In $(A)$, the statistical significance was assessed with a one-way ANOVA test, whereas, in $(C-D)$, a Student's unpaired $t$ test was used. 


\section{Blocking CYP2E1 Activity in AEII Cells Limits TG Accumulation}

Although the liver is predominantly responsible for metabolizing $\mathrm{EtOH}$, the lung possesses similar enzymatic capacity. As shown in Figures 5A and 5B, ADH activity and the CYP2E1 enzyme are present in the lung and enhanced in response to $\mathrm{EtOH}$. Because byproducts of EtOH metabolism are largely responsible for mediating lipid accumulation in the liver, we sought to determine whether $\mathrm{EtOH}$ or one of its metabolites is most important in promoting TG synthesis in L2 cells.

In contrast to whole lung, $\mathrm{ADH}$ activity was not detected in L2 cells cultured in media alone or in media supplemented with $\mathrm{EtOH}$ (data not shown). However, expression of the CYP2E1 enzyme was readily identified in L2 cells, and levels were significantly increased in response to $\mathrm{EtOH}$ (Figure 5C). To assess whether this enzymatic activity is important in promoting metabolic alterations in L2 cells, we examined the effects of EtOH on TG levels in cells cultured in the presence or absence of chlormethiazole (CMZ), a CYP2E1 inhibitor. Pretreatment with CMZ $(100 \mu \mathrm{M})$ for 24 hours completely prevented TG accumulation in EtOHtreated L2 cells (Figure 5D), indicating that products of EtOH metabolism are involved in TG accumulation after EtOH exposure. Interestingly, treating L2 cells with the antioxidant, $\mathrm{N}$-acetylcysteine $(5 \mathrm{mM})$, for 24 hours failed to attenuate $\mathrm{EtOH}$-induced TG accumulation, suggesting that, at least under these experimental conditions, lipid accumulation may not depend upon the generation of reactive oxygen species
(Figure 5E). However, we recognized that antioxidant effects of $\mathrm{N}$-acetylcysteine were not directly monitored in these studies.

\section{Chronic Alcohol Exposure Alters Macrophage Phenotype in the Lung} Because $\mathrm{EtOH}$ is known to alter AM behavior, we sought to evaluate the effects of chronic EtOH consumption on AM phenotypes in our model system. Consistent with prior reports, we detected a twofold increase in mRNA content of Tgf-b1 in freshly isolated primary AMs from lungs of $\mathrm{EtOH}$-fed rats (Figure 6A), and this was associated with a nonsignificant increase in TGF- $\beta 1$ protein in BAL fluid (Figure 6B). The increased Tgf-bl expression was associated with enhanced expression of Arg1 (Figure 6A), but not Tnf- $\alpha$ transcripts after chronic EtOH feeding. Together, this

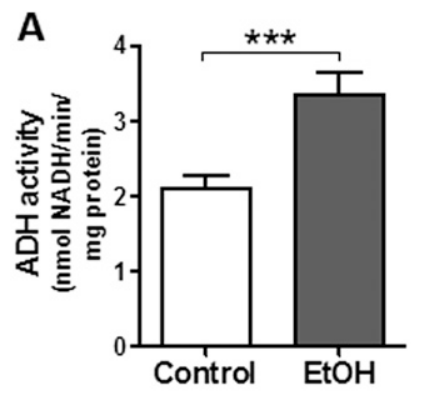

B

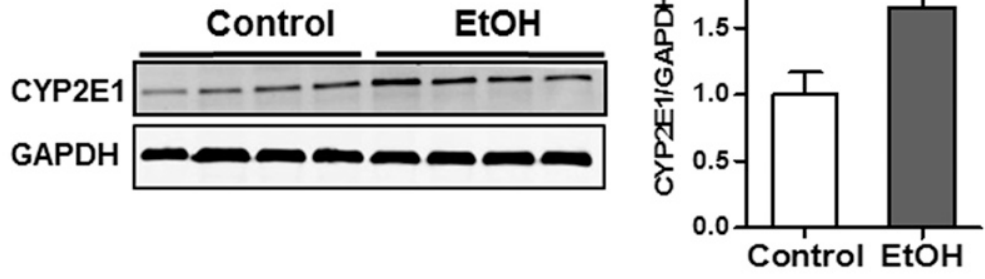

C
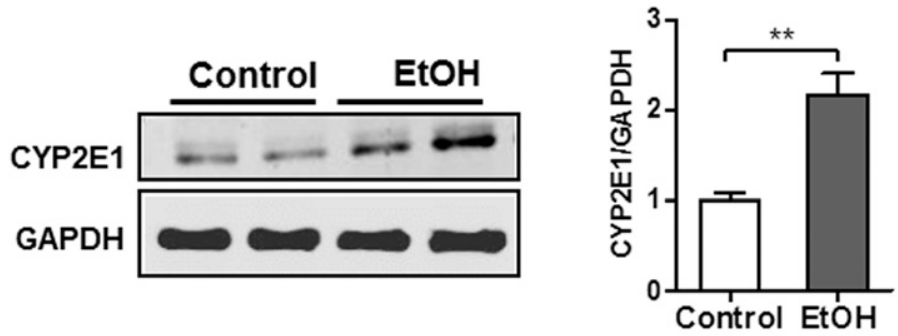

D

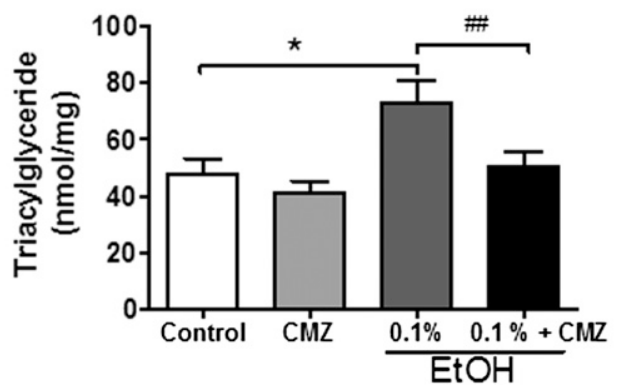

E

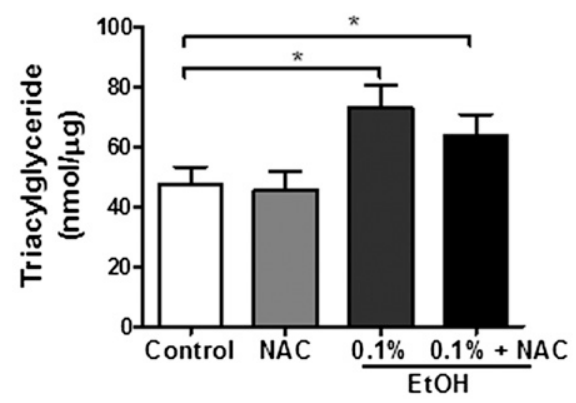

Figure 5. Inhibition of CYP2E1 activity blocks TG accumulation in AEll cells (A). Alcohol dehydrogenase (ADH) activity is detected in the lung and activity increases in response to chronic alcohol consumption $\left(n=6,{ }^{\star \star \star} P<0.001\right.$ versus control group). (B) EtOH induces protein expression of $\mathrm{CYP} 2 \mathrm{E} 1$ in the rat lung ( $n=6,{ }^{*} P<0.05$ versus control group). The image is representative of three different blots. (C) EtOH induces protein expression of $\mathrm{CYP} 2 \mathrm{E} 1$ in rat L2 AEll cells $\left(n=6,{ }^{\star \star} P<0.01\right.$ versus control group). (D) Treatment of L2 cells with the CYP2E1 inhibitor chlormethiazole (CMZ) for 24 hours blockedx EtOH-induced TG accumulation ( $n=6,{ }^{\star} P<0.05$ versus control group, ${ }^{\# \#} P<0.01$ versus EtOH group). (E) The antioxidant $\mathrm{N}$-acetyl cysteine did not attenuate EtOH-induced TG accumulation in L2 cells $\left(n=6,{ }^{\star} P<0.05\right.$ versus control group). Data are expressed as means ( $\pm \mathrm{SE}$ ). In (A-C), the statistical significance was assessed using a Student's unpaired $t$ test, whereas, in $(D-E)$, a one-way ANOVA test was used. 

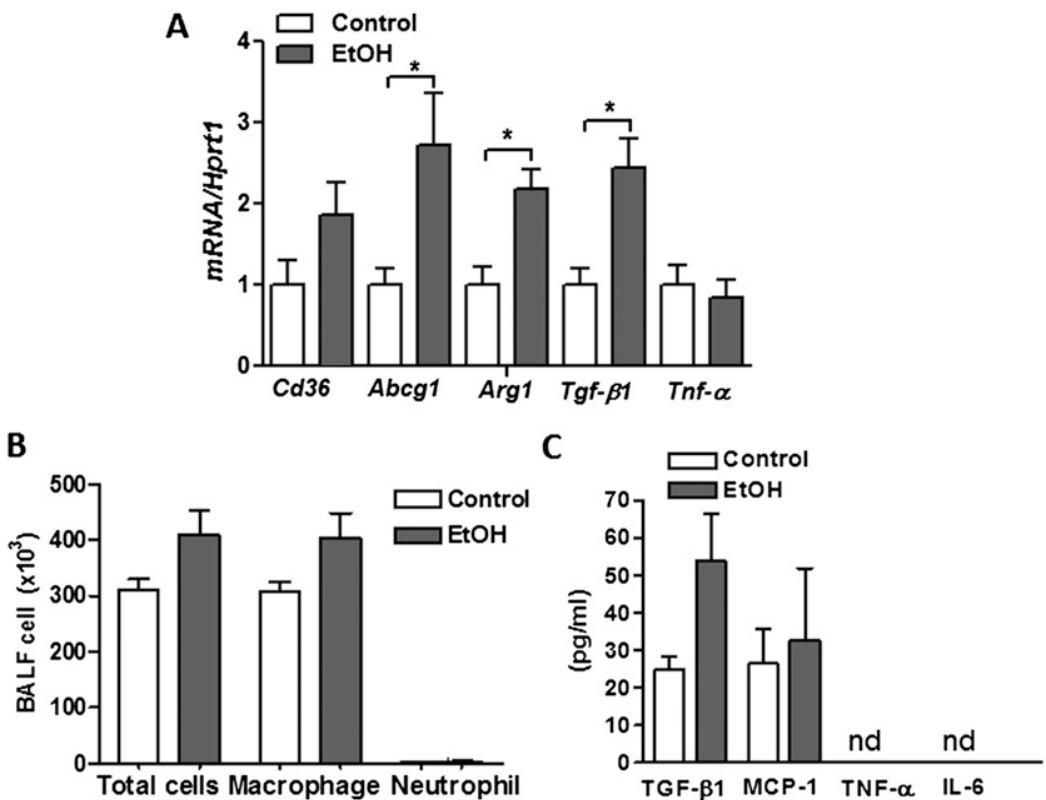

Figure 6. Chronic EtOH exposure alters primary AM phenotype. (A) Transcript levels for Cd36, ATPbinding cassette sub-family $\mathrm{G}$ member 1 (Abcg1), agrinase-1 (Arg1), transforming growth factor-1 (Tgfb1), and Tnf- $\alpha$ in freshly isolated AMs from lungs of EtOH-fed rats. (B) Total and differential cell counts in BAL fluid from control and EtOH-fed rats. (C) ELISA for TGF- $\beta 1$, monocyte chemoattractant protein (MCP)-1, TNF- $\alpha$, and IL-6 in BAL fluid from control and EtOH-fed rats. Data are expressed as means ( \pm SE). The statistical significance was assessed using a Student's unpaired t test. $\mathrm{n}=6,{ }^{\star} P<0.01$ versus control group.

gene expression profile suggested that chronic EtOH intake polarizes AMs toward an $\mathrm{M} 2$ reparative phenotype. Interestingly, AMs isolated from lungs of EtOH-fed rats also displayed higher mRNA expression of the lipid receptor, $C d 36$, as well as the lipid transporter, Abcg1, which presumably serves as an adaptive response to the increased extracellular lipids (Figure 6A). Despite these changes in AM gene expression, we did not observe a significant increase in the total number of AMs recovered from BAL fluid or in levels of the inflammatory markers monocyte chemoattractant protein 1, TNF- $\alpha$, or IL-6 in BAL fluid from EtOH-fed rats. The latter observation may reflect low expression levels of some of these factors (i.e., TNF- $\alpha$ and IL-6), as well as a dilutional effect of lavage, rendering some factors undetectable in our experiments (Figure 6B).

\section{FAs Promote M2 Polarization and Exacerbate the Suppressive Effects of Alcohol on AM Function}

Because chronic EtOH consumption was associated with changes in AM gene expression, we hypothesized that lipid accumulation within distal airspaces might contribute to these findings. To test this hypothesis, we examined the effect of the FA palmitate on AM phenotype in culture. Consistent with findings in AMs isolated from EtOH-exposed lungs, rat AMs cultured in media containing $250 \mu \mathrm{M}$ of palmitic acid displayed increased mRNA expression of lipid receptor, $C d 36$, and lipid efflux transporters, Abca1 and Abcg1 (Figure 7A). Moreover, palmitic acid promoted a shift in macrophage phenotype to an $\mathrm{M} 2$ reparative subtype characterized by increased mRNA expression of Arg1 and $T g f b 1$ and decreased expression of the M1 marker, Tnf- $\alpha$ (Figure 7B).

To determine whether these phenotypic changes were associated with functional impairments, we assessed the phagocytic capacity of AMs cultured in the presence or absence of palmitic acid. As shown in Figure 7C, palmitic acid significantly reduced uptake of heat-killed $E$. coli by AMs. Moreover, this functional deficit was equivalent to that observed with alcohol treatments, and was additive when cells were cultured with alcohol plus palmitate. Furthermore, palmitic acid blunted agonistinduced inflammatory responses in AM, decreasing both TNF- $\alpha$ and IL- 6 production in response to LPS (Figures $7 \mathrm{D}$ and $7 \mathrm{E}$ ). Taken together, these findings indicate that extracellular FA accumulation alters AM phenotype and function, and suggest that metabolic abnormalities may be mechanistically linked to immune impairments in the alcoholic lung.

\section{Discussion}

Chronic EtOH abuse predisposes to the development of inflammatory lung diseases, and several mechanisms have been proposed to explain these clinical observations. These include altered oxidant-antioxidant balance $(24,25)$ and direct suppressive effects on AMs $(26,27)$. In this study, we describe a mechanism in which EtOH impairs lung lipid homeostasis, associated with altered activation of AMPK, with the net effects of enhanced lipid synthesis and impaired immune function. We show that EtOH induces marked lipid accumulation in the lung, a pathological phenotype reminiscent of the alcoholic fatty liver, and we provide data implicating these lipids in the functional impairment of AMs.

The major lipid species that were increased in lungs from chronic EtOH-fed rats were TGs and FAs. The functional importance of these lipids in the adult lung has not been fully determined, although it is believed that TGs and FAs contribute to the fluidity of the surfactant monolayer and provide FA acyl chains for PL production $(15,28)$. For the latter reason, and because we observed enhanced expression of the lipid-synthesizing machinery (e.g., Fasn) in response to $\mathrm{EtOH}$, we were surprised to find that PL levels were not significantly altered in response to chronic EtOH consumption. However, we postulate that the lung has evolved protective mechanisms to limit fluctuations in PL levels that might otherwise imperil pulmonary function. This hypothesis is consistent with reports showing decreased PL synthesis in AEII cells after an acute EtOH exposure, but stable PL levels after chronic EtOH ingestion $(18,19)$.

Although we did not observe significant changes in total PL levels in response to chronic EtOH feeding, we recognize that our findings do not exclude the possibility that EtOH might have altered the type of PLs produced. In the liver, chronic EtOH ingestion significantly disrupts hepatic desaturase activity, leading to marked 

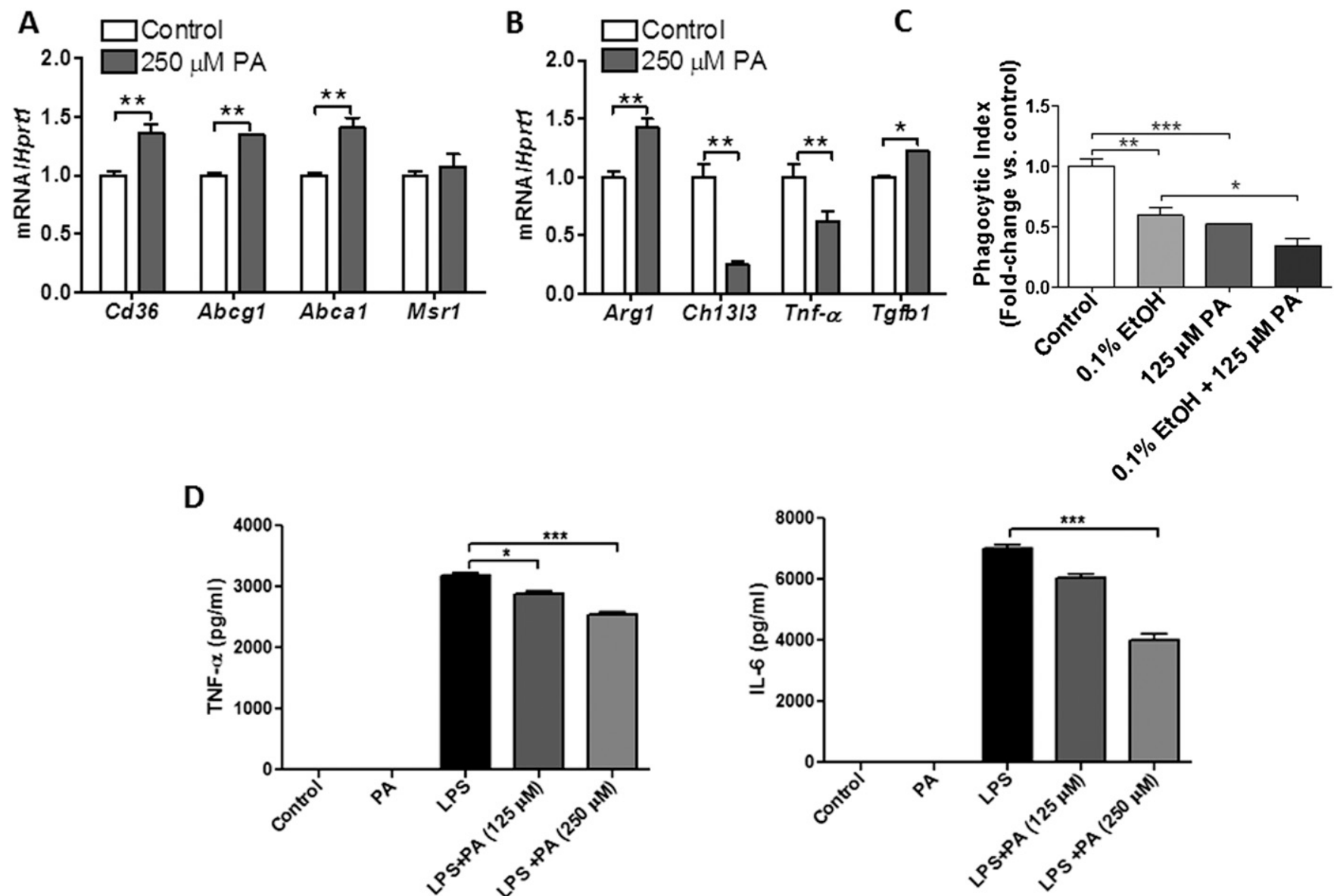

Figure 7. Increases in FAs alter AM phenotype and function. (A) Rat AMs cultured in media containing $250 \mu M$ of palmitic acid (PA) displayed an increased mRNA expression of lipid receptor, Cd36, and the lipid efflux transporters, Abca1 and Abcg1 ( $n=6$, ${ }^{\star \star} P<0.01$ versus control group). (B) PA promotes a shift toward an M2 macrophage phenotype in rat AMs, characterized by enhanced mRNA expression or Arg1 and Tgfb1 and suppression of Tnf- $\alpha$ transcripts $\left(n=6,{ }^{\star} P<0.05\right.$, ${ }^{\star \star} P<0.01$ versus control group). (C) PA significantly reduced uptake of heat-killed $E$. coli by AMs, and this effect was enhanced with $\mathrm{EtOH}$ treatment $\left(n=6,{ }^{\star \star} P<0.01,{ }^{\star \star \star} P<0.001\right.$ versus control group and ${ }^{\star} P<0.05$ versus EtOH group). (D) PA blunts LPSinduced proinflammatory responses in rat AMs $\left(n=6,{ }^{*} P<0.05\right.$, ${ }^{\star *} P<0.001$ versus control group). Data are expressed as means ( \pm SE). The statistical significance was assessed using a one-way ANOVA test.

changes in the intrahepatic fatty acyl-chain composition (29). Similar biochemical changes in the lung might have important consequences; recent studies have demonstrated that genetic mutations leading to deranged FA composition in the lung result in enhanced fibroproliferative responses, as well as altered pulmonary compliance $(30,31)$.

Our findings are consistent with a model in which EtOH-induced accumulation of pulmonary TG and FA results from increased lipid synthesis in AEII cells. This is based on our findings showing that several key factors (e.g., SREBF1, DGAT1) involved in TG synthesis are up-regulated in the alcohol-exposed lung (32). Most notably, we detected a marked increase in the transcription factor, Srebf1, in both whole lung and in cultured AEII cells. Recent evidence indicates that Srebf1 plays an important role in regulating TG levels in the lung (33). Mice with deletions of insulin-induced gene 1 and insulin-induced gene 2, both of which encode for proteins that inhibit Srebf, displayed enhanced Srebf1 function associated with marked accumulation of TGs in both AEII cells and AMs (32, 34, 35). Conversely, deletion of the sterolregulatory-element-binding cleavageassociated protein, which is required for Srebf activation, was associated with a nonsignificant decrease in TG levels in AEII cells. These findings suggest that the effects of EtOH on Srebf1 might contribute to lipid accumulation in affected lungs (36).

Another mechanism by which EtOH might contribute to lipid accumulation in the lung is by inhibiting the breakdown of FAs (37). Importantly, this mechanism has been shown to play a critical role in lipid accumulation in the alcoholic liver (38). Consistent with this possibility, we found that Ppara and Cpt1a mRNA expression were significantly decreased in the EtOH-exposed rat lung. Our observation that similar decreases in Ppara and Cpt1a expression were not seen in cultured AEII cells in response to EtOH suggests that TG accumulation in our model systems may depend more upon enhanced production than upon reduced elimination of excess lipids.

We observed that chronic EtOH ingestion inhibits AMPK activation in the lung. Decreased AMPK phosphorylation was demonstrated in both whole-lung tissue and in cultured AEII cells after exposure 
to $\mathrm{EtOH}$. AMPK is a central regulator of metabolism, and might reasonably be expected to participate in the metabolic defects seen in the EtOH-exposed lung $(39,40)$. Consistent with this hypothesis, decreased AMPK has been shown to promote alcoholic fatty liver disease, and pharmacological activation of AMPK has been shown to limit alcohol-induced steatosis in mice $(41,42)$. Such observations provide a rationale for testing whether activators of AMPK have similar effects on lipid homeostasis in the lung.

The ability of the lung to metabolize $\mathrm{EtOH}$ indicates that deleterious effects can be induced by either $\mathrm{EtOH}$ or one of its metabolites. In this study, we demonstrated that TG accumulation in AEII cells is largely dependent on the ability of AEII cells to metabolize EtOH; inhibition of the CYP2E1 enzyme with CMZ completely abolished TG accumulation (43). We believe that these findings may have important clinical implications, because drugs that inhibit the cytochrome enzymes are clinically available. Whether inhibition of CYP2E1 can prevent the toxic effects of EtOH on pulmonary cells in our animal models or in humans remains to be studied.

One important complication of alcoholic liver disease is the development of respiratory insufficiency. This is explained by various factors, including ascites, hepatic hydrothorax, as well as the enigmatic condition known as hepato-pulmonary syndrome. Although arterial hypoxemia in patients with liver disease is often attributed to diverse mechanisms (i.e., lung restriction, vascular shunts), we hypothesize that lipid accumulation within the distal airspaces might also play a role. This hypothesis is supported by the observation that TG accumulation in the lungs of male Zucker diabetic fatty rats is associated with capillary basement membrane thickening and impairments in gas exchange $(44,45)$. Future studies examining the relationship between lung lipid abnormalities and the development of respiratory insufficiency in alcoholic patients are warranted.

Our observations also suggest a mechanistic link between metabolic abnormalities and immune impairments in the lung. To date, the majority of studies focusing on immune dysregulation have investigated the direct effects of EtOH on immune cell function (46-48). In this study, we propose an alternative hypothesis, namely, that chronic EtOH exposure of AEII cells stimulates a paracrine lipid excess wherein FAs released into the distal air spaces are concentrated by macrophages, promoting an alternative macrophage phenotype and impairing macrophage function. Ongoing studies will test whether antilipid therapies might prevent and/or treat EtOH-related inflammatory lung disorders.

In summary, we found that chronic alcohol exposure alters lung metabolic homeostasis, and our data suggest that these abnormalities might play a role in the development of inflammatory lung diseases. We anticipate that these findings will open new avenues of research regarding the effects of alcohol on lung biology, and will provide a foundation for future clinical investigations examining the role of metabolic changes in the development of diverse lung diseases.

Author disclosures are available with the text of this article at www.atsjournals.org.

\section{References}

1. Perlino CA, Rimland D. Alcoholism, leukopenia, and pneumococcal sepsis. Am Rev Respir Dis 1985;132:757-760.

2. Cook RT. Alcohol abuse, alcoholism, and damage to the immune system-a review. Alcohol Clin Exp Res 1998;22: 1927-1942.

3. Zhang P, Bagby GJ, Happel KI, Summer WR, Nelson S. Pulmonary host defenses and alcohol. Front Biosci 2002;7:d1314-d1330.

4. Polikandriotis JA, Rupnow HL, Elms SC, Clempus RE, Campbell DJ, Sutliff RL, Brown LA, Guidot DM, Hart CM. Chronic ethanol ingestion increases superoxide production and NADPH oxidase expression in the lung. Am J Respir Cell Mol Biol 2006;34:314-319.

5. Brown SD, Brown LA. Ethanol (EtOH)-induced TGF- $\beta 1$ and reactive oxygen species production are necessary for EtOH-induced alveolar macrophage dysfunction and induction of alternative activation. Alcohol Clin Exp Res 2012;36:1952-1962.

6. Moss M, Bucher B, Moore FA, Moore EE, Parsons PE. The role of chronic alcohol abuse in the development of acute respiratory distress syndrome in adults. JAMA 1996;275:50-54.

7. Berkowitz DM, Danai PA, Eaton S, Moss M, Martin GS. Alcohol abuse enhances pulmonary edema in acute respiratory distress syndrome. Alcohol Clin Exp Res 2009;33:1690-1696.

8. Moss M, Burnham EL. Chronic alcohol abuse, acute respiratory distress syndrome, and multiple organ dysfunction. Crit Care Med 2003; 31(4 suppl):S207-S212.

9. Joshi PC, Guidot DM. The alcoholic lung: epidemiology, pathophysiology, and potential therapies. Am J Physiol Lung Cell Mol Physiol 2007;292: L813-L823.

10. Mitchell PO, Jensen JS, Ritzenthaler JD, Roman J, Pelaez A, Guidot DM. Alcohol primes the airway for increased interleukin-13 signaling. Alcohol Clin Exp Res 2009;33:505-513.

11. Zhong W, Cui Y, Yu Q, Xie X, Liu Y, Wei M, Ci X, Peng L. Modulation of LPS-stimulated pulmonary inflammation by borneol in murine acute lung injury model. Inflammation 2014;37:1148-1157.
12. O'Shea RS, Dasarathy S, McCullough AJ; Practice Guideline Committee of the American Association for the Study of Liver Diseases; Practice Parameters Committee of the American College of Gastroenterology. Alcoholic liver disease. Hepatology 2010;51:307-328.

13. Menon KV, Gores GJ, Shah VH. Pathogenesis, diagnosis, and treatment of alcoholic liver disease. Mayo Clin Proc 2001;76:1021-1029.

14. Lee M, Kowdley KV. Alcohol's effect on other chronic liver diseases. Clin Liver Dis 2012;16:827-837.

15. Whitsett JA, Wert SE, Weaver TE. Alveolar surfactant homeostasis and the pathogenesis of pulmonary disease. Annu Rev Med 2010;61:105-119.

16. Mason RJ. Surfactant synthesis, secretion, and function in alveoli and small airways: review of the physiologic basis for pharmacologic intervention. Respiration 1987;51:3-9.

17. Liau DF, Hashim SA, Pierson RN III, Ryan SF. Alcohol-induced lipid change in the lung. $J$ Lipid Res 1981;22:680-686.

18. Holguin F, Moss I, Brown LA, Guidot DM. Chronic ethanol ingestion impairs alveolar type II cell glutathione homeostasis and function and predisposes to endotoxin-mediated acute edematous lung injury in rats. J Clin Invest 1998;101:761-768.

19. Wagner M, Heinemann HO. Effect of ethanol on phospholipid metabolism by the rat lung. Am J Physiol 1975;229:1316-1320.

20. Lang CH, Derdak Z, Wands JR. Strain-dependent differences for suppression of insulin-stimulated glucose uptake in skeletal and cardiac muscle by ethanol. Alcohol Clin Exp Res 2014;38:897-910.

21. Shah D, Romero F, Stafstrom W, Duong M, Summer R. Extracellular ATP mediates the late phase of neutrophil recruitment to the lung in murine models of acute lung injury. Am J Physiol Lung Cell Mol Physiol 2014;306:L152-L161.

22. Iverson SJ, Lang SL, Cooper MH. Comparison of the Bligh and Dyer and Folch methods for total lipid determination in a broad range of marine tissue. Lipids 2001;36:1283-1287.

23. Viollet B, Mounier R, Leclerc J, Yazigi A, Foretz M, Andreelli F. Targeting AMP-activated protein kinase as a novel therapeutic approach for the treatment of metabolic disorders. Diabetes Metab 2007;33:395-402. 
24. Liang Y, Harris FL, Jones DP, Brown LA. Alcohol induces mitochondrial redox imbalance in alveolar macrophages. Free Radic Biol Med 2013;65:1427-1434.

25. Jensen JS, Fan X, Guidot DM. Alcohol causes alveolar epithelial oxidative stress by inhibiting the nuclear factor (erythroid-derived 2)like 2-antioxidant response element signaling pathway. Am J Respir Cell Mol Biol 2013;48:511-517.

26. Thevenot P, Saravia J, Giaimo J, Happel KI, Dugas TR, Cormier SA. Chronic alcohol induces M2 polarization enhancing pulmonary disease caused by exposure to particulate air pollution. Alcohol Clin Exp Res 2013;37:1910-1919.

27. Curry-McCoy TV, Venado A, Guidot DM, Joshi PC. Alcohol ingestion disrupts alveolar epithelial barrier function by activation of macrophagederived transforming growth factor beta1. Respir Res 2013;14:39.

28. Agassandian M, Mallampalli RK. Surfactant phospholipid metabolism. Biochim Biophys Acta 2013;1831:612-625.

29. Umeki S, Shiojiri H, Nozawa Y. Chronic ethanol administration decreases fatty acyl-CoA desaturase activities in rat liver microsomes. FEBS Lett 1984;169:274-278.

30. Sunaga $H$, Matsui $H$, Ueno M, Maeno T, Iso T, Syamsunarno MR, Anjo S, Matsuzaka T, Shimano H, Yokoyama T, et al. Deranged fatty acid composition causes pulmonary fibrosis in Elovl6-deficient mice. Nat Commun 2013;4:2563.

31. Goetzman ES, Alcorn JF, Bharathi SS, Uppala R, McHugh KJ, Kosmider B, Chen R, Zuo YY, Beck ME, McKinney RW, et al. Longchain acyl-CoA dehydrogenase deficiency as a cause of pulmonary surfactant dysfunction. J Biol Chem 2014;289:10668-10679.

32. Plantier L, Besnard V, Xu Y, Ikegami M, Wert SE, Hunt AN, Postle AD, Whitsett JA. Activation of sterol-response element-binding proteins (SREBP) in alveolar type II cells enhances lipogenesis causing pulmonary lipotoxicity. J Biol Chem 2012;287:10099-10114.

33. Zhang F, Pan T, Nielsen LD, Mason RJ. Lipogenesis in fetal rat lung: importance of C/EBPalpha, SREBP-1c, and stearoyl-CoA desaturase. Am J Respir Cell Mol Biol 2004;30:174-183.

34. Shimano $\mathrm{H}$. Sterol regulatory element-binding proteins (SREBPs): transcriptional regulators of lipid synthetic genes. Prog Lipid Res 2001;40:439-452.

35. Besnard V, Wert SE, Stahlman MT, Postle AD, Xu Y, Ikegami M, Whitsett JA. Deletion of Scap in alveolar type II cells influences lung lipid homeostasis and identifies a compensatory role for pulmonary lipofibroblasts. J Biol Chem 2009;284:4018-4030.

36. You M, Fischer M, Deeg MA, Crabb DW. Ethanol induces fatty acid synthesis pathways by activation of sterol regulatory elementbinding protein (SREBP). J Biol Chem 2002;277:29342-29347.
37. Sozio M, Crabb DW. Alcohol and lipid metabolism. Am J Physiol Endocrinol Metab 2008;295:E10-E16.

38. Crabb DW. Alcohol deranges hepatic lipid metabolism via altered transcriptional regulation. Trans Am Clin Climatol Assoc 2004;115: 273-287.

39. Jian MY, Alexeyev MF, Wolkowicz PE, Zmijewski JW, Creighton JR. Metformin-stimulated AMPK- $\alpha 1$ promotes microvascular repair in acute lung injury. Am J Physiol Lung Cell Mol Physiol 2013;305: L844-L855.

40. Zhao X, Zmijewski JW, Lorne E, Liu G, Park YJ, Tsuruta Y, Abraham E. Activation of AMPK attenuates neutrophil proinflammatory activity and decreases the severity of acute lung injury. Am J Physiol Lung Cell Mol Physiol 2008;295:L497-L504.

41. García-Villafranca J, Guillén A, Castro J. Ethanol consumption impairs regulation of fatty acid metabolism by decreasing the activity of AMP-activated protein kinase in rat liver. Biochimie 2008;90: 460-466.

42. Ben Mosbah I, Massip-Salcedo M, Fernández-Monteiro I, Xaus C, Bartrons R, Boillot O, Roselló-Catafau J, Peralta C. Addition of adenosine monophosphate-activated protein kinase activators to University of Wisconsin solution: a way of protecting rat steatotic livers. Liver Transp/ 2007;13:410-425.

43. Correa M, Viaggi C, Escrig MA, Pascual M, Guerri C, Vaglini F, Aragon $\mathrm{CM}$, Corsini GU. Ethanol intake and ethanol-induced locomotion and locomotor sensitization in Cyp2e1 knockout mice. Pharmacogenet Genomics 2009;19:217-225.

44. Foster DJ, Ravikumar $\mathrm{P}$, Bellotto DJ, Unger $\mathrm{RH}$, Hsia CC. Fatty diabetic lung: altered alveolar structure and surfactant protein expression. Am J Physiol Lung Cell Mol Physiol 2010;298:L392-L403.

45. Farkas GA, Schlenker EH. Pulmonary ventilation and mechanics in morbidly obese Zucker rats. Am J Respir Crit Care Med 1994;150: 356-362.

46. Chen MM, Bird MD, Zahs A, Deburghgraeve C, Posnik B, Davis CS, Kovacs EJ. Pulmonary inflammation after ethanol exposure and burn injury is attenuated in the absence of IL-6. Alcohol 2013;47: 223-229.

47. Bird MD, Zahs A, Deburghgraeve C, Ramirez L, Choudhry MA, Kovacs EJ. Decreased pulmonary inflammation following ethanol and burn injury in mice deficient in TLR4 but not TLR2 signaling. Alcohol Clin Exp Res 2010;34:1733-1741.

48. Standiford TJ, Danforth JM. Ethanol feeding inhibits proinflammatory cytokine expression from murine alveolar macrophages ex vivo. Alcohol Clin Exp Res 1997;21:1212-1217. 